\title{
From Requisite to Right: Assessing and Addressing Paid Maternity Leave in US Psychiatry Residency Programs
}

\author{
Rachel L. Dillinger ${ }^{1}$
}

Received: 30 July 2020 / Accepted: 10 August 2021 / Published online: 24 September 2021

(c) Academic Psychiatry 2021

\begin{abstract}
Objective Data on the physical and mental health benefits of paid maternity leave for mothers and infants is abundant. Data on the make-up of current maternity leave policies in US psychiatry residency programs is not. This survey of program directors was undertaken to assess the components of their program's policies and the perceived impact of maternity leave on the training of childbearing residents, co-residents, and programs.

Methods An anonymous 19-question survey was emailed to US psychiatric residency program directors. Questions assessed demographics for respondents and their programs, composition of maternity leave including paid and unpaid components, and the perception of effects of maternity leave on childbearing residents, co-residents, and programs (with optional freetext elaboration).

Results The response rate was $19.5 \%$ (49 out of 262 program directors). Many programs require the use of FMLA (81\%), vacation days (75\%), sick days (75\%), and short-term disability (30\%) for maternity leave. Around a third (34\%) offer separate paid time off varying from 2 to 12 weeks at $80-100 \%$ of pay. Most respondents relate a neutral to strongly positive impact of leave on the psychiatric training of childbearing residents (98\%) and co-residents (84\%), citing benefits like improved empathy, compassion, and patience.

Conclusions Maternity leave is seen to have minimal negative impact on training received within psychiatry residency programs. A minority of residents have access to paid maternity leave policies that would best support their health and career trajectories.
\end{abstract}

Keywords Paid maternity leave $\cdot$ Psychiatry residency programs $\cdot$ Maternity leave policies

Two decades ago, the International Labor Organization established a minimum global standard of 18 weeks paid maternity leave [1]. Of 42 developed nations, 7 fail to meet these recommendations [1]. Six of those offer 14-17 weeks at $80-100 \%$ of base salary [1]. This leaves the USA, offering 12 weeks of unpaid leave, the only developed country to not mandate paid maternity leave [1].

For infants, paid maternity leave is correlated with improved health outcomes including decreased mortality, increased childhood immunization rates, and increased rates of exclusive breastfeeding [2-4]. For mothers, paid leave

Rachel L. Dillinger

rdillinger@som.umaryland.edu

1 University of Maryland School of Medicine/Sheppard Pratt, Baltimore, MD, USA is also associated with improved health outcomes: it offers time to recover physically and supports breastfeeding, which in turn decreases risk of breast and ovarian cancers and is associated with longer lifespan $[2,5]$. The risk of postpartum depression is lowered for those taking paid maternity leave, especially with durations greater than 12 weeks $[1,2,6]$.

Graduate medical education surveys reveal $27-40 \%$ of women plan to have children during training $[5,7]$. The average medical graduate is 27 years old ( $15 \%$ over 30$)$, which corresponds with a slight decline in fertility that becomes more marked at age 35 [7]. Studies show female physicians typically have their first child 7.4 years after the general population [8]. In one study, $24 \%$ of female physicians were diagnosed with infertility; a fifth ultimately were unable to conceive [8]. Postponing pregnancy until training completion may result in pregnancy complications, unintentional childlessness, and having fewer children than desired [7]. 
Still, many female residents fear that having children will extend training, jeopardize fellowship positions, and interfere with career plans [7]. In one study, trainees with children were $23 \%$ less likely to consider a career in academic medicine than those without children; reasons included concerns around work-life balance and difficulty identifying role models who had achieved having both families and career success [9]. Women comprise just $19 \%$ of full professors, $13 \%$ of department chairs, and $11 \%$ of deans [10]. This paucity of women at the highest ranks of academic medicine conveys to female trainees that they must choose between their personal life and career advancement and reveals missed opportunities to recruit and retain top physician leaders, educators, and researchers [10].

With the likelihood of residents planning to conceive, their well-documented concerns, and the growing body of literature supporting the benefits of paid leave, psychiatry residency program directors face a challenge. Broadly, they must ensure staffing of core clinical services which limits flexibility, particularly at smaller programs and those with many female trainees $[5,11]$. They must consider equity for co-residents and heed eligibility requirements for the Accreditation Council for Graduate Medical Education (ACGME) and American Board of Psychiatry and Neurology (ABPN) board certification. Extensions in training can delay certification exams and complicate funding, as most financial support for residents comes from Medicare (limited to 48 months) [5, 11]. Guidance from the ACGME psychiatry program requirements vaguely stipulates that each program must allow "an appropriate length of absence" for residents taking parental leave [12].

There is scant literature about maternity leave policies in US psychiatry residency programs. This paper reports the results of a survey of general adult psychiatry program directors designed to investigate these policies along with the views of program directors on the impact of maternity leave on training for the childbearing trainee, co-residents, and overall program.

\section{Methods}

An anonymous 19-question survey was designed for US adult psychiatry residency program directors regarding maternity leave policies and the perceived impact on childbearing trainees and their co-residents when maternity leave was taken. The survey included limited demographic questions (respondent gender, geographic region, and program size) to enable assessment of representation and trends. Other questions assessed for percentage of female trainees, existence and components of maternity leave policies including paid leave, and the perceived impact of taking maternity leave on the childbearing resident, co-residents, and program (with the option to elaborate further with free-text responses).

The survey was declared not human subjects research and determined to not require review by the University of Maryland School of Medicine IRB. It was sent via email to program directors in April 2020. A reminder email was sent 2 weeks later, and the survey closed 1 week after that. Quantitative data was analyzed using IBM SPSS version 25.0 (Armonk, NY) via ratio calculations, chi-squared analysis, and nonparametric cross-tabulation. Qualitative data via free-text responses was examined using content analysis with themes described in the "Results" section.

\section{Results}

Of the emails sent to the 262 listed program directors, 7 returned as "undeliverable," 4 replies stated they were not program directors and requested to be removed, 2 replies were automated "out of office" messages, and 3 replies were automated generic program information. Conservatively, if the message made it to the 5 that generated automatic replies, the response rate for the survey was 19.5\% (49/251).

In terms of policy composition, almost all respondents $(95.9 \%, 47 / 49)$ reported their programs had an official maternity leave policy. Figure 1 displays the various components of maternity leave that residents had access to, along with the duration of any separate paid maternity leave offered.

At programs where most residents were female, paid leave was more likely to be available (1.45:1 with $>50 \%$ female, $1: 1$ with $\leq 50 \%$ female). There were no associations found between the presence of paid leave being offered and program size (0.6:1 for small, 1.66:1 at medium, 0.8:1 at large), program director gender $\left(\chi^{2}=0.328, p=0.567\right)$, or geography (due to low numbers of programs in Southwest (4) and West (4), chi-squared assumptions were violated and the likelihood ratio was 8.26 with a $p=0.082$ ).

Fewer program directors at large ( $11+$ residents per class $)$ programs reported that past residents who took maternity leave have had to extend their training (0.8:1) compared to those at smaller (10 or fewer residents per class) programs (3.5:1). Most programs (33/49) noted past residents extended training. No association was found between extension of training and program director gender $\left(\chi^{2}=0.428\right.$, $p=0.807$ ) or geographical region (assumptions were violated due to multiple expected values less than 5 , likelihood ratio of 6.150 with $p=0.630$ ).

Program director views on the impact of taking maternity leave on training for the childbearing resident and their coresidents can be seen in Fig. 2. A theme of growth emerged in free-text responses regarding childbearing residents taking leave, citing that "becoming a parent is a maturing experience that deepens psychotherapy abilities" and "parenting/ 
Fig. 1 Percent of programs offering different maternity leave components and duration of separate paid maternity leave with percentages of programs offering it

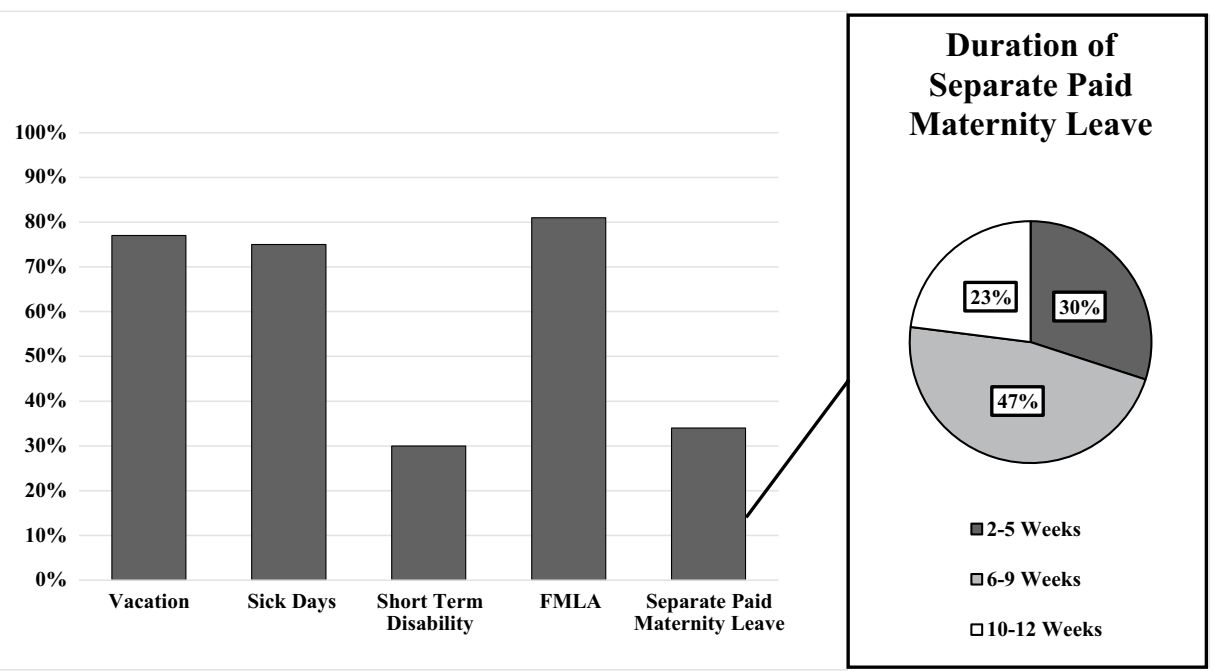

caretaking contributes to specific psychiatric skills and increases empathy, compassion and patience." For maternity leave effects on co-residents, a theme of culture and support appeared frequently. Illustrative responses include "the birth of children is celebrated in our program," "encouraging leave promotes wellness and focus on work-life balance," and "co-residents are supportive and step up without issue." For the minority reporting negative effects on co-residents, responses touched on resentfulness and the need for service coverage.

Geography did appear to have an association with the perception of impact of maternity leave on co-residents' training (assumptions violated as above, but with likelihood ratio of 22.042 and $p=0.037$ ). The view of it having a positive effect was more than predicted in the Southwest and less than predicted in the West. The view of it having a negative effect was more than predicted in the Northeast and Midwest, and less than predicted in the Southeast.

The theme of barriers captured many of the concerns program directors reported related to the effect of a resident taking maternity leave on the program. Challenges involved

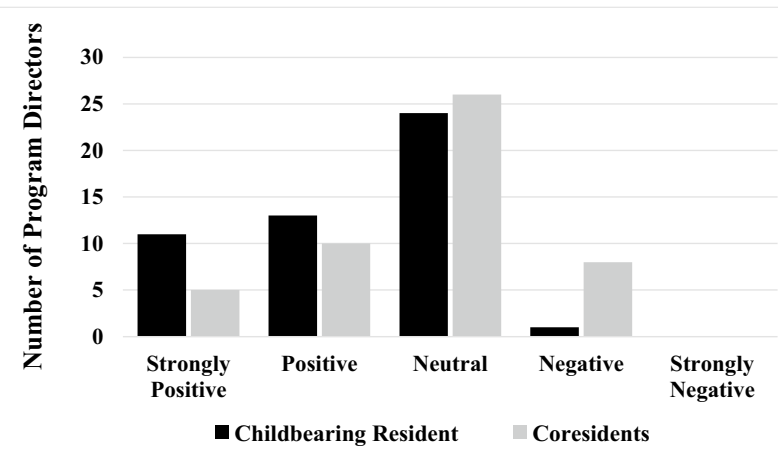

Fig. 2 Program director perception of the impact of taking maternity leave on the training of childbearing resident and co-residents arranging service coverage, creating and funding off-cycle positions, and coordinating required experiences. Other problems included understanding complicated legal aspects and board requirements, lack of Family and Medical Leave Act coverage in intern year resulting in less leave or leave without pay, and feeling constrained by state or institutional policies.

Finally, program directors offered creative solutions for transitioning back to work and protecting pay while maximizing time to heal and bond. These included scholarly work (research, reading, study of child development and perinatal mental health) and remote work (made easier by telehealth and the SARS-CoV-2 pandemic). Additional arrangements included lighter rotations the month of projected delivery and return, working part time, avoiding night float, and ensuring a slow reintegration to the call schedule.

\section{Discussion}

Although none of the responding programs offers a maternity leave policy commensurate with the International Labor Organization guidelines, 24\% (12/49) offer separate paid leave in line with task force recommendations [5, 11]. Over a third (34\%) offer paid leave separate from other contributions, which is promising. Unfortunately, this leaves most programs that responded with policies that literature shows are detrimental to the financial, physical, and mental health of women and their children $[1,2,5,6]$. The vast majority of program directors viewed the effect on training for both the childbearing resident and their co-residents as neutral to positive, which may help alleviate trainee concerns.

While representation concerns were somewhat mitigated for geography/program director gender/etc., results were subject to nonresponse and voluntary response bias, where those who did respond may feel compelled by this 
topic and have more generous policies while the opposite may be true for those who declined to respond. Responses were anonymous to help encourage honest answers, but social desirability bias may have altered responses. Together, these biases may result in a falsely favorable picture of the current state of policies. The chief limitation of this study was the survey response rate, which limits generalizability and resulted in subgroup analyses having too few numbers to identify and calculate statistically significant associations.

A higher response rate, combined with future research on maternal and infant outcomes-specifically in childbearing residents - could help strengthen the case for paid leave. However, current results reflect identification of the need to enact multi-level change to standardize paid maternity leave policies that are congruent with other developed nations, particularly in the absence of state and federal legislation.

The most immediate opportunity for change is at the programmatic level. Program directors can move beyond the current mandate to simply provide a maternity leave policy to residents and offer explicit information regarding schedule adjustments, available opportunities, and required rotations; this may also alleviate co-resident concerns around equity and division of labor [11].

ABPN eligibility requirements currently stipulate that the 4 weeks minimum per year of vacation should be averaged over 4 years [13]. This policy allows both for more than 4 weeks per year to be taken without affecting board eligibility and allows a trainee who knows they are going to give birth in the following year to allocate one or more weeks from the current year, for example.

There is time to make up experiences and maximize postpartum healing and bonding, a notion supported by a study of US psychiatric residency programs revealing $100 \%$ had 2-12 months of PGY-4 elective time [14]. ACGME guidelines require scholarly endeavors and stipulate that electives must have a "written curriculum with goals and objectives, and learning experiences that lead to specific learning outcomes" [12]. Opportunities to apply those requirements to meet the needs of new mothers include comprehensive reading lists, reflective writing, weekly meetings with mentors, summative projects, and telecommuting clinical electives [15]. Respondents to this survey employ these options along with offering part-time return (permitted by the ABPN provided it is $\geq 50 \%$ [13]), slow reintegration into the call schedule, and inclusion of electives in child development and reproductive psychopharmacology (both explicitly tested on the ABPN exam) [16]. For those fast tracking into child and adolescent psychiatry (CAP) fellowship, both the ACGME and ABPN allow for up to 12 months of certain core requirements and experiences to count towards both adult and CAP requirements $[12,13]$.
Targets for change at the institution level include adding FMLA-style protections for trainees in their first 12 months and providing full salaries during leave $[5,11]$. Providing staffing via moonlighting, locums, or service attendings transiently absorbing resident workload would alleviate coresident burden and concerns of equity, guilt, and resentment [11]. Finally, institutions should provide access to and/ or funding for affordable, convenient childcare and backup care [11].

At the ABPN level, their governing body (American Board of Medical Specialties) recently stated that as of July 1, 2021, member boards will allow 6 weeks of leave during training "without exhausting time allowed for vacation or sick leave and without requiring an extension in training" [17]. One such member board, the American Board of Obstetrics and Gynecology, allows a total of 6 months leave over the 4-year training period [18]. The pediatric board allows for extended leave without prolonging graduation if the trainee is "well qualified and has met all the training requirements" [19]. The addition of similar language to the psychiatry document would help alleviate concerns surrounding extended training and fellowship and related concerns about funding and off-cycle positions. This would also support the shift from time-based to competency-based medical education [11].

An impactful change at the ACGME level would be strengthening the current psychiatry document wording to mandate a stated minimum length of paid leave. Prior task forces recommend that the ACGME mandate paid maternity leave, starting at 6-8 weeks and working up to 12 weeks $[5,11]$. This would supersede inadequate institutional and program policies.

The results of this study support that many-but not enough-programs are trying to work around barriers to create policies to support residents and show that childbearing can enrich their careers and abilities, not simply pose a threat to them. Additional multilevel support is needed.

\section{Declarations}

Disclosures The author states that there is no conflict of interest.

Ethical Considerations The University of Maryland School of Medicine IRB determined that the submission did not require IRB review.

\section{References}

1. Burtle A, Bezruchka S. Population health and paid parental leave: what the United States can learn from two decades of research. Healthcare. 2016. https://doi.org/10.3390/healthcare4020030. 
2. Heymann J, Sprague AR, Nandi A, Earle A, Batra P, Schickedanz A, Chung PJ, Raub A. Paid parental leave and family wellbeing in the sustainable development era. Public Health Rev. 2017. https:// doi.org/10.1186/s40985-017-0067-2.

3. Rossin M. The effects of maternity leave on children's birth and infant health outcomes in the United States. J Health Econ. 2011. https://doi.org/10.1016/j.jhealeco.2011.01.005.

4. Thakrar AP, Forrest AD, Maltenfort MG, Forrest CB. Child mortality in the US and 19 OECD comparator nations: a 50-year timetrend analysis. Health Aff. 2018. https://doi.org/10.1377/hlthaff. 2017.0767.

5. Vassallo P, Jeremiah J, Forman L, Dubois L, Simmons DL, Chretien K, Amin A, Coleman D, Collichio F. Parental leave in graduate medical education: recommendations for reform. Am J Med. 2019. https://doi.org/10.1016/j.amjmed.2018.11.006.

6. Andres E, Baird S, Bingenheimer JB, Markus AR. Maternity leave access and health: a systematic narrative review and conceptual framework development. Matern Child Health J. 2016. https://doi. org/10.1007/s10995-015-1905-9.

7. Willett LL, Wellons MF, Hartig JR, Roenigk L, Panda M, Dearinger AT, Allison J, Houston TK. Do women residents delay childbearing due to perceived career threats? Acad Med. 2010. https:// doi.org/10.1097/ACM.0b013e3181d2cb5b.

8. Stentz NC, Griffith KA, Perkins E, Jones RD, Jagsi R. Fertility and childbearing among American female physicians. Journal of Women's Health. 2016. https://doi.org/10.1089/jwh.2015.5638.

9. Edmunds LD, Ovseiko P v., Shepperd S, Greenhalgh T, Frith P, Roberts NW, Pololi LH, Buchan AM (2016) Why do women choose or reject careers in academic medicine? A narrative review of empirical evidence. The Lancet. https://doi.org/10.1016/S01406736(15)01091-0

10. Valantine H, Sandborg CI. Changing the culture of academic medicine to eliminate the gender leadership gap: $50 / 50$ by 2020 . Acad Med. 2013. https://doi.org/10.1097/ACM.0b013e3182a34952.

11. Weinstein DF, Mangurian C, Jagsi R. Parenting during graduate medical training - practical policy solutions to promote change. N Engl J Med. 2019. https://doi.org/10.1056/NEJMp1904721.
12. Accreditation Council for Graduate Medical Education. Program requirements for graduate medical education in psychiatry. 2019. Available from: https://www.acgme.org/Portals/0/PFAssets/Progr amRequirements/400_Psychiatry_2019.pdf?ver=2019-08-26134127-827. Accessed 1 April 2020

13. American Board of Psychiatry and Neurology. 2020 information for applicants initial certification examination in psychiatry. 2019. Available from: https://www.abpn.com/wp-content/uploads/2019/ 10/2020_Psychiatry_CERT_Information_for_Applicants.program directorf. Accessed 1 April 2020.

14. Vestal HS, Belitsky R, Bernstein CA, et al. Required and elective experiences during the 4th year: an analysis of ACGME accredited psychiatry residency program websites. Acad Psychiatry. 2016. https://doi.org/10.1007/s40596-016-0507-1.

15. Webb AMB, Hasty BN, Andolsek KM, Mechaber HF, Harris TB, Chatterjee A, Lautenberger DM, Gottlieb AS. A timely problem: parental leave during medical training. Acad Med. 2019. https:// doi.org/10.1097/ACM.0000000000002733.

16. American Board of Psychiatry and Neurology. Certification examination in psychiatry 2020 content outline. 2019. Available from: https://www.abpn.com/wp-content/uploads/2019/10/2020_Psych iatry_CERT_Content_Specifications.program directorf. Accessed 1 April 2020.

17. American Board of Medical Specialties. ABMS announces progressive leave policy for residents and fellows. 2020. Available from: https://www.abms.org/news-events/abms-announces-progr essive-leave-policy-for-residents-and-fellows/\#ParentalLeave. Accessed 17 June 2020.

18. American Board of Obstetrics and Gynecology. Residency leave policy. 2019. Available from: https://www.abog.org/about-abog/ policies/residency-leave-policy. Accessed 4 April 2020.

19. The American Board of Pediatrics. General pediatrics training irregularities. 2020. Available from: https://www.abp.org/content/ general-pediatrics-training-irregularities. Accessed 17 June 2020

Publisher's Note Springer Nature remains neutral with regard to jurisdictional claims in published maps and institutional affiliations. 